\title{
Search for Top and Bottom Squarks
}

\author{
Carsten Rott \\ (for the ALEPH, CDF, DELPHI, L3, and OPAL Collaborations) \\ Purdue University, West Lafayette, Indiana 47907, USA, e-mail: carott@physics.purdue.edu
}

October 6, 2003

\begin{abstract}
Searches for the lightest scalar top quark $\tilde{t}_{1}$ and scalar bottom quark $\tilde{b}_{1}$ performed at LEP2 with a center-of-mass energy of up to $\sqrt{s}=209 \mathrm{GeV}$ and at Tevatron using data collected at $\sqrt{s}=1.8 \mathrm{TeV}$ during Run I and at $\sqrt{s}=1.96 \mathrm{TeV}$ during Run II, are discussed. Different possible decay modes were considered and no evidence for any such signal was observed. Exclusion limits were extracted.
\end{abstract}

PACS. 14.80.Ly Supersymmetric partners of known particles

\section{Introduction}

Supersymmetric (SUSY) extensions of the Standard Model (SM) predict for each SM particle the existence of a SUSY partner. The states $\tilde{q}_{L}$ and $\tilde{q}_{R}$ are the partners of the lefthanded and right-handed quarks. They form squark mass eigenstates $\left(\tilde{q}_{1}\right.$ and $\left.\tilde{q}_{2}\right)$, which are orthogonal combinations of them. The mixing angle $\theta_{\tilde{q}}$ is defined in such a way that $\tilde{q}_{1}=\tilde{q}_{L} \cos \left(\theta_{\tilde{q}}\right)+\tilde{q}_{R} \sin \left(\theta_{\tilde{q}}\right)$ is the lighter squark. Since the off-diagonal elements of the mass matrix are proportional to the mass of the corresponding SM partner, $m_{q}\left(A_{q}-\mu \kappa\right)$, the mixing is expected to be relevant for the fermions of the third family. In that expression $\mu$ is the Higgs mass parameter, $A_{q}$ the trillinear coupling to the Higgs sector and $\kappa=\tan \beta$ for down-type quarks and $\kappa=\frac{1}{\tan \beta}$ for up-type quarks. Therefore the sbottom quark could be light if $\tan \beta$, the ratio of the vacuum expectation values of the two Higgs doublet fields, is large. A light stop could be realized due to large mass splitting due to the large top mass.

\section{Searches for third generation squarks}

In this section the direct searches for squarks of the third generation performed at LEP and Tevatron are described. In R-parity conserving scenarios SUSY particles are pair produced and the lightest supersymmetric particle (LSP) is stable. R-parity $R_{p}=(-1)^{2 S+3 B+L}$ is a discrete, multiplicative symmetry defined by the spin $S$, baryon number $\mathrm{B}$, and lepton number $L$ of the particle. For the searches reported here, $R_{p}$ is conserved unless explicitly stated otherwise. At hadron colliders stops or sbottoms would be produced via the strong interaction, while at lepton colliders electroweakly. The coupling of the stop and sbottom to the $\mathrm{Z}$ depends on the mixing angle $\theta_{\tilde{t}}$, and $\theta_{\tilde{b}}$ respectively. Hence, at lepton colliders the production cross-section is not only a function of the squark mass, but also of the corresponding mixing angle. This coupling and therefore the production cross-section are maximal for $\theta_{\tilde{t}}=0^{\circ}$. The stop decouples from the $\mathrm{Z}$ for $\theta_{\tilde{t}}=56^{\circ}$ (similar for the sbottom for $\theta_{\tilde{b}}=68^{\circ}$ ) and the production cross-section is minimal.

\subsection{Searches for top squarks}

The decay $\tilde{t}_{1} \rightarrow t \tilde{\chi}_{1}^{0}$ is expected to be dominant if kinematically allowed. Due to the large top mass and limits on $\tilde{\chi}_{1}^{0}$, this mode is currently experimentally not accessible and is therefore not considered.

Then the three-body decay $\tilde{t}_{1} \rightarrow b l \tilde{\nu}$ via chargino would become the dominant decay mode if kinematically allowed. This yields two acoplanar jets, two acoplanar leptons and missing energy. Searches for this decay mode were performed at LEP2 and at the Tevatron. In $107 \mathrm{pb}^{-1}$ of CDF Run I data [1] and $108 \mathrm{pb}^{-1}$ of $\mathrm{D} \emptyset$ Run I data [2], no evidence for the existence of stop was found and exclusion limits were set. The results are shown in Figure 1 and compared to the combined limit from the LEP2 experiments [3]. If the chargino is Higgsino-like, the branching fraction of the decay $\tilde{t}_{1} \rightarrow b \tau \tilde{\nu}$ is considerably enhanced. For this case ALEPH [4], L3 [5] and OPAL [6] obtained limits slightly below or similar to those for requiring similar branching ratios for all leptonic species.

If the three-body decay is not allowed kinematically, then the loop suppressed flavor changing two body decay $\tilde{t}_{1} \rightarrow c \tilde{\chi}_{1}^{0}$ is expected to be the dominant decay channel. This decay results in events with two acoplanar jets and missing energy. Searches for the two body decay in the non-degenerate state (Mass degenerate and nearly mass degenerate searches are discussed in section 2.3) of the stop were performed ALEPH [7], DELPHI [8], L3 [5], and OPAL [6]. Table 1 summarizes some of the results obtained in these searches. 


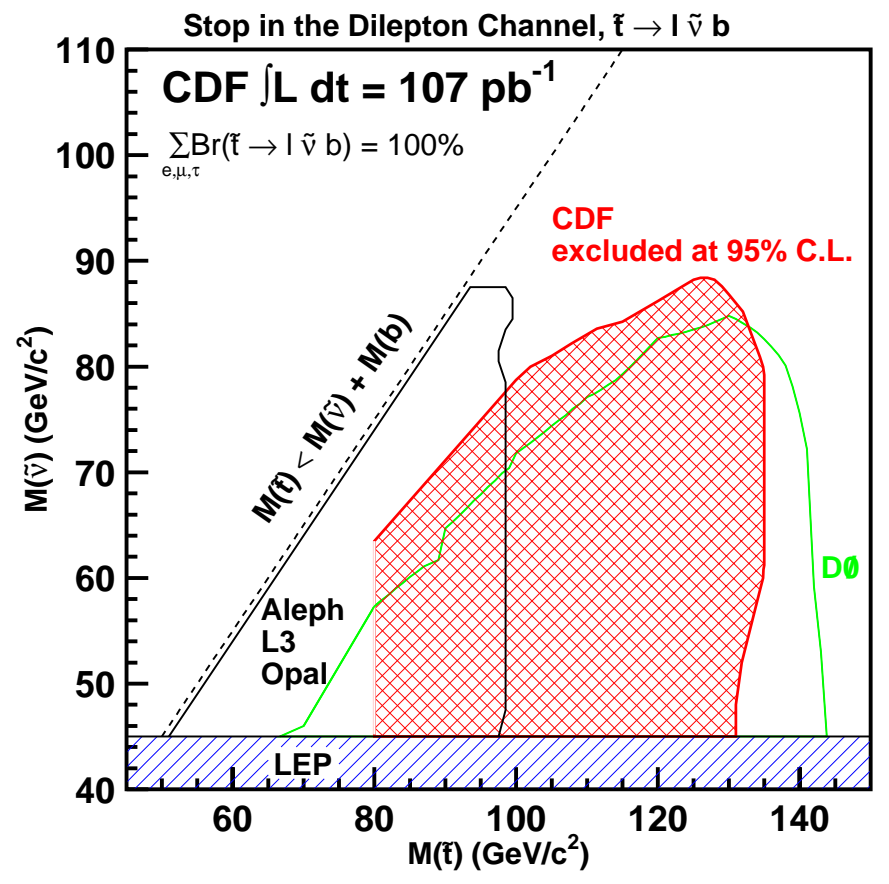

Fig. 1. Stop and sneutrino mass plane showing the CDF $95 \%$ C.L. excluded region as hatched area compared to D $\varnothing$ and LEP2, for the three-body stop decay, $\tilde{t}_{1} \rightarrow l \tilde{\nu} b$.

Table 1. Limits given here are for the decay $\tilde{t}_{1} \rightarrow c \tilde{\chi}_{1}^{0}$ for the case in which $\tilde{t}_{1}$ decouples from the Z. $\Delta m$ is defined as $\Delta m=m_{\tilde{t}_{1}}-m_{\tilde{\chi}_{1}^{0}}$.

\begin{tabular}{lll}
\hline Experiment & $\Delta m\left[\mathrm{GeV} / c^{2}\right]$ & Limit $\left[\mathrm{GeV} / c^{2}\right]$ \\
\hline ALEPH & $>8$ & 92 \\
DELPHI & $>10$ & 92 \\
L3 & $15-25$ & 90 \\
OPAL & $>10$ & 95.7 \\
\hline
\end{tabular}

Stop could also decay in a four-body decay mediated by virtual chargino and $\mathrm{W}$ or virtual chargino and sfermion to $\tilde{t}_{1} \rightarrow b \tilde{\chi}_{1}^{0} f \tilde{f}^{\prime}$. The presence of jets, b-jets, leptons and missing energy is the signature for this decay. A multi-jet analysis was performed at ALEPH [4] that was combined with the acoplanar jet analysis (two body decay). This allows constraints to be set under the more general hypothesis that both the $\tilde{t}_{1} \rightarrow b \tilde{\chi}_{1}^{0} f \bar{f}^{\prime}$ and $\tilde{t}_{1} \rightarrow c \tilde{\chi}_{1}^{0}$ decay channels contribute to that stop decay and the results are shown in Figure 2.

An $R_{p}$ violating analysis was performed with $106 \mathrm{pb}^{-1}$ of CDF Run I data. It was assumed stops are pair produced and then decay $\tilde{t}_{1} \rightarrow b \tau$, with one of the $\tau$ 's sequentially decaying hadronically and the second leptonically. A stop upper mass limit of $122 \mathrm{GeV} / c^{2}$ was set [9].

\subsection{Searches for bottom squarks}

Sbottom quarks $\tilde{b}_{1}$ are expected to decay into $b \tilde{\chi}_{1}^{0}$, yielding two acoplanar jets and missing energy. Searches in this

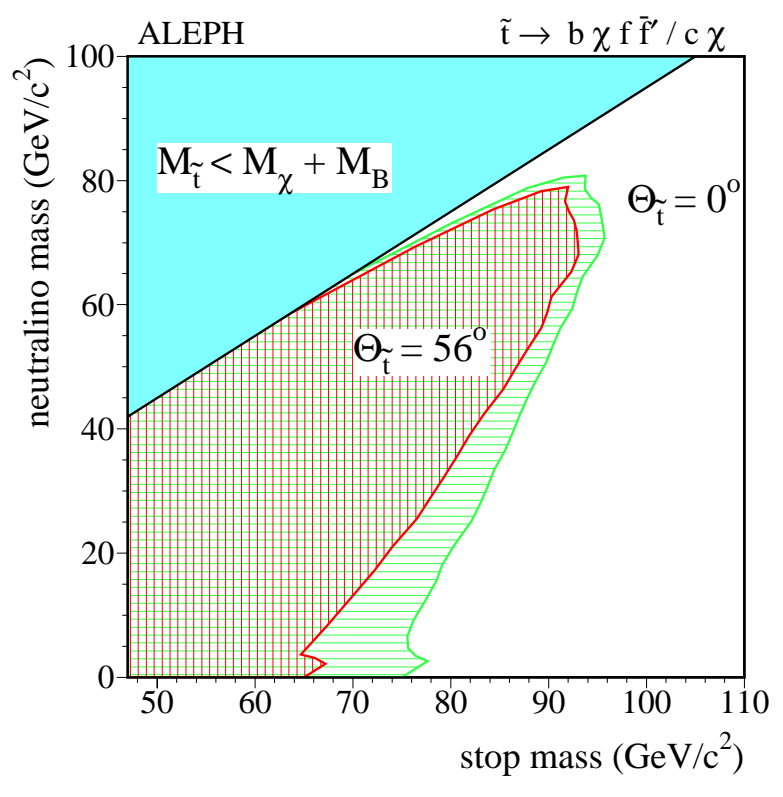

Fig. 2. Branching ratio independent exclusion region at $95 \%$ C.L. in the $m_{\tilde{t}_{1}}$ vs. $m_{\tilde{\chi}_{1}^{0}}$ plane, from $\tilde{t}_{1} \rightarrow b \tilde{\chi}_{1}^{0} f \bar{f}^{\prime}$ and $\tilde{t}_{1} \rightarrow c \tilde{\chi}_{1}^{0}$ searches.

decay channel follow an approach similar to the one in the stop two body decay. In the case of the $\tilde{b}_{1}$ analyses b-tagging of jets is often required. Figure 3 compares the limit obtained by OPAL [6] with the CDF Run I [10] limit (which used b-tagging). ALEPH [4], DELPHI [8], and L3 [5] which used b-tagging obtain exclusion limits similar to OPAL.

The sbottom pair production cross-section is small compared to the gluino pair production cross-section at hadron colliders. If the sbottom is lighter than the gluino, the decay $\tilde{g} \rightarrow \tilde{b}_{1} \bar{b}$ is expected to be dominant. In a sequential decay the sbottom would then decay to $\tilde{b}_{1} \rightarrow b \tilde{\chi}_{1}^{0}$. Sbottom quarks from gluino decays yield a very distinctive signature of 4 b-jets and missing energy. A search for sbottom quarks from gluino decays was performed with $38.4 \mathrm{pb}^{-1}$ of CDF Run II data. In the preselection, events were selected with three jets $\left(E_{T}>15 \mathrm{GeV}\right.$ and $|\eta|<2)$ and missing transverse energy $E_{T}>35 \mathrm{GeV}$. Specific cuts were applied to reduce contributions from the QCD background. The signal region was defined by $\not_{T}>50 \mathrm{GeV}$ and by vetoing events containing isolated leptons, to reduce the background from $t \bar{t}, \mathrm{~W}+\mathrm{jets}$, and $\mathrm{Z}$ + jets. In addition, one or two b-tagged jets were required. b-jets were identified using a secondary vertex tagging algorithm. Tracks with large impact parameter were selected and a vertex fitting algorithm was used to reconstruct a displaced vertex. In the signal region four events were observed with a single b-tag, which is in good agreement with the predicted SM contribution of $5.6 \pm 1.4$ events. Similarly, one double b-tag event was observed with $0.5 \pm 0.1$ expected. No evidence for sbottom quarks from gluino de- 


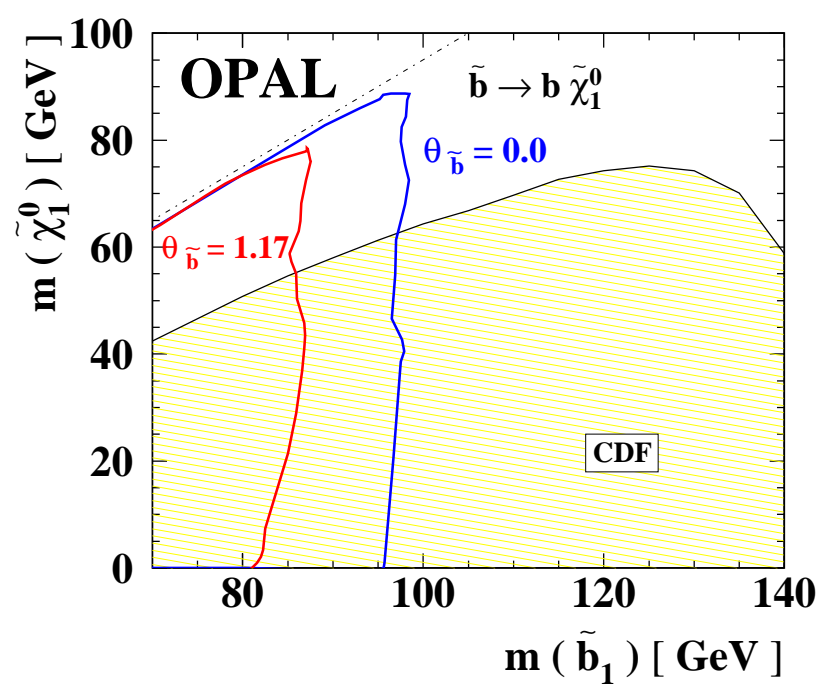

Fig. 3. $95 \%$ C.L. excluded regions in the $\left(m_{\tilde{b}_{1}}, m_{\tilde{\chi}_{1}^{0}}\right)$ plane assuming that $\tilde{b}_{1}$ decays into $b \tilde{\chi}_{1}^{0}$. The solid (dotted) line shows for maximal (minimal) coupling of the $\tilde{b}_{1}$ to the $\mathrm{Z}$ the exclusion limits obtained by OPAL. The singly-hatched regions are excluded by CDF Run I data.

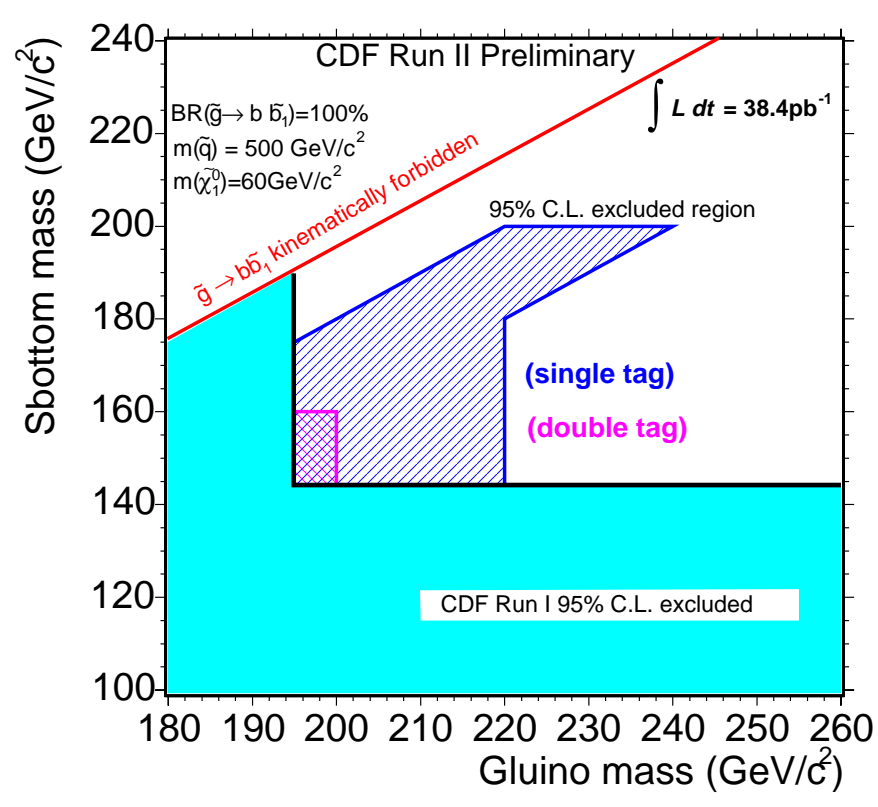

Fig. 4. $95 \%$ C.L. contour exclusion in the plane $\left(m_{\tilde{g}}, m_{\tilde{b}_{1}}\right)$ for $\left.m_{\tilde{\chi}_{1}^{0}}=60 \mathrm{GeV} / c^{2}\right)$ as obtained by requiring a single b-tag or a double b-tag.

cays was found and preliminary exclusion limits for the $\left(m_{\tilde{g}}, m_{\tilde{b}_{1}}\right)$ plane were extracted. Figure 4 shows the contour exclusion obtained by this search assuming a neutralino mass of $60 \mathrm{GeV} / c^{2}$.

\subsection{Search for long lived particles}

The decay width of the stop two body decay $\tilde{t}_{1} \rightarrow c \tilde{\chi}_{1}^{0}$ is proportional to $m_{\tilde{t}_{1}}\left(1-m_{\tilde{\chi}_{1}^{0}}^{2} / m_{\tilde{t}_{1}}^{2}\right)^{2}$. Hence, for the nearly mass degenerate case, for $\Delta m=m_{\tilde{t}_{1}}-m_{\tilde{\chi}_{1}^{0}} \leq$ $6 \mathrm{GeV} / c^{2}$ the lifetime of the stop becomes sizable and must be taken into account. If $\Delta m$ is so small that $\tilde{t}_{1} \rightarrow c \tilde{\chi}_{1}^{0}$ is kinematically closed then $\tilde{t}_{1} \rightarrow u \tilde{\chi}_{1}^{0}$ becomes the dominant decay channel and the stop can be considered stable for experimental purposes. Searches for stable and metastable hadrons were performed by ALEPH [11] and a lower stop mass limit of $63 \mathrm{GeV} / c^{2}$ was obtained. In a search for stable squarks DELPHI [12] excluded $\tilde{t}$ in the mass range of $2-80 \mathrm{GeV} / c^{2}$ and $\tilde{b}$ for the range $5-38 \mathrm{GeV} / c^{2}$. A search for charged massive stable particles was conducted using $53 \mathrm{pb}^{-1}$ CDF Run II data, making use of CDF's time of flight detector [13]. Events were selected with an isolated high transverse momentum, a long time of flight, and an anomalously large ionization energy loss (indication of a slow moving heavy particle). Seven events were observed with $2.9 \pm 0.8$ (stat) \pm 3.1 (sys) expected events from SM background, allowing a preliminary limit of $108 \mathrm{GeV} / c^{2}$ to be set on the mass of a stable stop quark.

\section{Conclusions}

Searches for pair production of stop and sbottom quarks were performed at LEP2 and Tevatron. No evidence for any such signal was observed. The results of the analyses were used to set limits on SUSY particles. The most recent results for stop and sbottom searches have been summarized, but a complete review can not be given here, for this I refer to the given references.

\section{References}

1. CDF Collaboration, Phys. Rev. Lett. 90, 251801 (2003)

2. D $\varnothing$ Collaboration, Phys. Rev. Lett. 88, 171802 (2002)

3. LEP SUSY Working Group, ALEPH, DELPHI,L3, and OPAL Collaborations, LEPSUSYWG/02-02.1 (2002), http://lepsusy.web.cern.ch/lepsusy/ and references therein.

4. ALEPH Collaboration, Phys. Lett. B 537 (2002) 5

5. L3 Collaboration, L3 Note 2804

6. OPAL Collaboration, Phys. Lett. B 545 (2002) 272 [Erratum-ibid. B 548 (2002) 258]

7. ALEPH Collaboration, Phys. Lett. 499 (2001) 67

8. DELPHI Collaboration, (http://delphiwww.cern.ch/ $\sim$ pubxx/delsec/conferences/summer03/PapNo094.html) CERN_EP2003-007. accepted by EPJ

9. CDF Collaboration, hep-ex/0305010. submitted to PRL

10. CDF Collaboration, Phys. Rev. Lett. 84, 5704 (2000)

11. ALEPH Collaboration, Phys. Lett. B 488, 234 (2000).

12. DELPHI Collaboration, Phys. Lett. B 444, 491 (1998)

13. CDF-II Collaboration, FERMILAB-PUB-96-390-E 